\section{Subcellular distribution and expression of cofilin and ezrin in human colon adenocarcino- ma cell lines with different metastatic potential}

\author{
D. Nowak, ${ }^{1}$ A.J. Mazur, ${ }^{1,2}$ \\ A. Popow-Woźniak, ${ }^{\prime}$ \\ A. Radwańska, ${ }^{1}$ H.G. Mannherz, ${ }^{2}$ \\ M. Malicka-Błaszkiewicz ${ }^{1}$
}

'Department of Cell Pathology, Faculty of Biotechnology, University of Wroctaw, Przybyszewskiego Wrocław, Poland; 2Department of Anatomy and Embryology, Ruhr-University, Bochum, Germany

\section{Abstract}

The dynamic reorganization of the actin cytoskeleton is regulated by a number of actin binding proteins (ABPs). Four human colon adenocarcinoma cell lines - parental and three selected sublines, which differ in motility and metastatic potential, were used to investigate the expression level and subcellular localization of selected ABPs. Our interest was focused on cofilin and ezrin. These proteins are essential for cell migration and adhesion. The data received for the three more motile adenocarcinoma sublines (EB3, 3LNLN, 5W) were compared with those obtained for the parental LS180 adenocarcinoma cells and fibroblastic NRK cells. Quantitative densitometric analysis and confocal fluorescence microscopy were used to examine the expression levels and subcellular distribution of the selected ABPs. Our data show distinct increase in the level of cofilin in adenocarcinoma cells accompanied by the reduction of inactive phosphorylated form of cofilin. In more motile cells, cofilin was accumulated at cellular periphery in co-localization with actin filaments. Furthemore, we indicated translocation of ezrin towards the cell periphery within more motile cells in comparison with NRK and parental adenocarcinoma cells.

In summary, our data indicate the correlation between migration ability of selected human colon adenocarcinoma sublines and subcellular distribution as well as the level of cofilin and ezrin. Therefore these proteins might be essential for the higher migratory activity of invasive tumor cells.

\section{Introduction}

In multicellular organisms only a few specialized cell types have the ability to migrate actively. During embryogenesis, a large number of cells or even sheets of coherent cells migrate throughout the process of organ formation or development of the nervous system. In an adult organism, only leukocytes and fibroblasts migrate during extravasation and wound healing, respectively. ${ }^{1}$ However, transformed malignant cells can regain the ability of active migration. ${ }^{2}$ Understanding of this process is therefore a fundamental part of cancer research. The basic difference between a benign and a malignant tumor is the ability of cancer cells to move. This process is called metastasis. The detailed comprehension of the cell migration process would lead to a better understanding of cellular mechanisms responsible for this pathological condition and possibly help in finding new approaches and tools for cancer treatment.

The actin cytoskeleton is a key structure in the regulation of cell motility, membrane trafficking, cell polarity and cytokinesis. ${ }^{1,3,4}$ To serve all these different requirements, cells need mechanisms to regulate the dynamics of actin filaments. Cellular activity and behaviour are regulated by external cues, which activate a number of small GTP-binding proteins of the Rho family. ${ }^{5}$ These regulate actin filament dynamics by coordinative activation of a number of actin binding proteins. ${ }^{6}$ Among them, the interaction of cofilin with monomeric and filamentous actin is very important, since it stimulates the dynamic behaviour of actin filaments. Cofilin severs and increases the rate of monomer dissociation from the minus-ends of filament, thus increasing the turnover of filamentous actin, especially at plasma membrane areas involved in cell protrusion. ${ }^{7 \cdot 13}$ The activity of cofilin is reversibly regulated by phosphorylation and dephosphorylation at Ser-3, with the phosphorylated form (P-cofilin) being inactive. It is known that LIM-kinase 1 (LIMK 1) and LIM-kinase 2 (LIMK 2), which activities are switched on by Rho-ROCK kinase and the corresponding phosphatase Slingshot, regulate cofilin activity by phosphorylation and dephosphorylation reactions. ${ }^{14,15}$

Ezrin, radixin and moesin (ERM family) are an important group of cytoplasmic ABPs involved in the organization of the submembranous actin cytoskeleton. The ERM proteins belong to the group of band 4.1 related proteins, which are proposed to function as membrane/cytoskeletal linkers..$^{16-19}$ The specific effect and action mechanism of this regulation are unclear, but many ABPs like the ERM proteins, which cellular distribution is close to the plasma membrane, are regulated by phospho-
Correspondence: Dr. Dorota Nowak, Department of Cell Pathology, Faculty of Biotechnology, University of Wrocław, Przybyszewskiego 63, 51-148 Wrocław, Poland.

Tel. +48.71.3756-289 - Fax: +48.71.3756-234

E-mail: dorotan@ibmb.uni.wroc.pl

Key words: actin, cofilin, ezrin, colon adenocarcinoma.

Acknowledgements: Dorota Nowak would like to thank the exchange program of the Ruhr- and Wroclaw Universities.

This work was partially supported by grant no. N N303 337535 from the Ministry of Science and Higher Education, Poland.

Received for publication: 28 December 2009. Accepted for publication: 1 February 2010.

This work is licensed under a Creative Commons Attribution 3.0 License (by-nc 3.0).

(C) Copyright D. Nowak et al., 2010

Licensee PAGEPress, Italy

European Journal of Histochemistry 2010; 54:e14 doi:10.4081/ejh.2010.e14

inositide lipids. Additionally, ezrin is phosphorylated by the Rho-dependent ROCK kinase ${ }^{20-22}$ and subsequently it is able to modulate actin cytoskeleton and to induce peripheral filopodia and microvilli. Indeed, it has been demonstrated that ezrin participates in cell adhesion, motility and cell survival. ${ }^{16,20,23,24}$ There is also increasing evidence that ezrin regulates tumor progression. ${ }^{23,25-28}$ Some data have indicated that this protein may be a key regulatory molecule in processes leading to tumor invasiveness. $^{29}$

In our previous studies, ${ }^{30,31}$ human colon adenocarcinoma LS180 cell sublines exhibiting an increased motility and metastatic potential ${ }^{32,33}$ were used to examine the possible correlation between the expression level of actin, in particular of $\beta$-actin, the state of actin polymerization and cell invasiveness. We found significant decrease in the level of monomeric actin and an increase in the state of actin polymerization as well as in $\beta$-actin expression in the cytosolic fraction of the three cell variants with a higher metastatic potential and invasiveness (EB3, 3LNLN, 5W) in comparison with the parental LS180 cell line. In this paper the same cellular model was used to investigate the levels and subcellular distribution of ABPs crucial for cell migration. The levels of expression and the subcellular localization of cofilin and ezrin were determined. The results obtained for the selected LS180 sublines were compared with those obtained for the parental colon adenocarcinoma line and normal rat kidney fibroblasts (NRK). 


\section{Materials and Methods}

\section{Cell culture}

The human colon adenocarcinoma cell line LS180 and the selected variants EB3, 3LNLN and $5 \mathrm{~W}$ were obtained from the Institute of Immunology and Experimental Therapy, Polish Academy of Sciences in Wroclaw. Originally the cell line LS180 came from Deutsche Krebsforschungzentrum, Heidelberg, Germany. The parental LS180 cell line and further selected sublines were propagated in OptiMEM medium supplemented with $3 \%$ fetal bovine serum (FBS) (Gibco, Grand Island, N.Y., USA). Cells were cultured in $25 \mathrm{~cm}^{2}$ tissue culture (TC) flasks (Falcon or Costar) at $37^{\circ} \mathrm{C}$ in $5 \% \mathrm{CO}_{2}$ 95\% humidified air and passaged twice a week, using $0.25 \%$ trypsin $/ 0.05 \%$ EDTA solution (Gibco, Grand Island, NY, USA). The details of the colon adenocarcinoma sublines selection procedures are described on following.

\section{In vitro selection of LS180 cells with increased affinity for HPLNEC.B3 cells}

The endothelial cell line HPLNEC.B3 human microvascular endothelial cells from a peripheral lymph node of a patient with Hodgkin's lymphoma - was isolated and characterized by Kieda et al..$^{33}$ HPLNEC.B3 cells were plated onto the bottom of 24 -well tissue culture plate. The colon adenocarcinoma LS180 cells $\left(1 \times 10^{5}\right.$ cells $\left./ 100 \mu \mathrm{L}\right)$ were superposed onto the upper surface of the $8 \mu \mathrm{m}$, Matrigel ${ }^{\circledast}$-coated nucleopore Transwell filter (Costar, France). After 48-72 h, the LS180 cells that migrated to the lower surface of the filter towards endothelial cell monolayer, were removed by a brief $0.25 \%$ trypsin/ $0.05 \%$ EDTA treatment, washed and propagated in vitro. The manipulation was repeated three times. The obtained colon cancer subline was named LS180EB3 (EB3).

\section{In vivo selection of colon adeno- carcinoma cells}

The in vitro selected EB3 cells were passaged in vivo by various routes of inoculation to select metastatic variants. For animal experiments, athymic $\mathrm{NCr}$ nu/nu male mice, obtained from National Cancer Institute, Frederic Cancer Research and Developmental Center (Frederick, MD, USA) were used. Animal experiments were performed according to the International Laboratory Animal Care Convention. The procedure of selection was applied essentially as described by Opolski $e t$ $a l .{ }^{32}$ Briefly, EB3 cells were injected intrasplenically (i.s.), resulting in primary tumors in the spleen and metastasis in the liver. Then a piece of liver metastasis was transplanted orthotopically into the intestinal wall (i.i.). The implanted tumors metastasized preferentially into the liver. The procedure was repeated five times resulting in a highly metastatic subline designed LS180EB3 5W (5W). A suspension of metastatic cells from the first i.s. passage was also injected intravenously (i.v.) and metastases in the lymph nodes were collected. In this case the passage was repeated three times, resulting in the variant designed LS180EB33LNLN (3LNLN), metastasizing after i.v. inoculation preferentially to peripheral lymph nodes.

Normal rat kidney fibroblasts (NRK) were obtained from the European Collection of Animal Cell Cultures. The cells were grown at $37^{\circ} \mathrm{C}$ and $5 \% \mathrm{CO}_{2}$ in plastic flasks in DMEM (Dulbecco's modified Eagle medium) supplemented with $10 \%$ FBS.

\section{Migration assay}

The day before the experiment cells were seeded into 6-well plates and starved for 6 hours in serum free DMEM medium. One-hundred $\mu \mathrm{L}$ of mixture 50\% Matrigel, 20\% FBS, 30\% OptiMEM, 5 nM Epidermal Growth Factor (EGF), used as chemoatractant, was poured at the bottom of 24-well plate and allowed to polymerize for $1 \mathrm{~h}$. Chemoatractant was then covered with $300 \mu \mathrm{L}$ of serum free DMEM. Next, rehydrated Transwell filters $(\varnothing 8 \mu \mathrm{m}$ pore size, BD Bioscienses) were placed in 24-well plate above the polymerized chemoatractant. Then cells were trypsinized, centrifuged $(800 \mathrm{xg}, 5$ min), resuspended in serum free DMEM and seeded onto Transwell filters in the number of $5 \times 10^{4}$. After $24 \mathrm{~h}$, the cells that did not migrate through the pores of the filter were precisely removed. The cells that migrated trough the filter were fixed (4\% paraformaldehyde in phosphate-buffered saline (PBS) for $20 \mathrm{~min}$ at room temperature), stained with Hoechst (Invitrogen) and counted. The migration factor [\%] was calculated as number of cells which migrated through the filter to the total number of seeded cells. The experiment was performed three times in nine repeats per every cell line.

\section{Adhesion assay}

The cell adhesion assay was performed as described by Humphries. ${ }^{34}$ Colon adenocarcinoma cells were seeded in triplicates onto 96well plate at the density of $1 \times 10^{4}$ cells per well. After $3 \mathrm{~h}$, the cells were rinsed with PBS, fixed with $4 \%$ paraformaldehyde in PBS for 20 minutes and nuclei were stained with Hoechst 32333. The numbers of adherent cells were counted under the fluorescence microscope at 200x magnification for each specimen. The adhesion factor [\%] was calculated as number of cells which adhered to plastic surface of cul- ture dish after 3 hours to the total number of cells seeded. The assay was performed three times for each cell line.

\section{Isolation of cytosolic fractions}

Cells were homogenized and the cytosolic fraction was prepared as described earlier by Malicka-Błaszkiewicz and Roth. ${ }^{35}$ Cells growing on tissue culture dishes were gently washed with PBS, scraped with a rubber policeman and suspended in monomeric actin stabilizing buffer (freshly made), containing $10 \mathrm{mM}$ Tris$\mathrm{HCl} \mathrm{pH} \mathrm{7.4;} \mathrm{0.25} \mathrm{M} \mathrm{sucrose;} 1 \mathrm{mM}$ DTT; $0.1 \mathrm{mM}$ ATP; $0.1 \mathrm{mM} \mathrm{CaCl}{ }_{2}$ (buffer A). Cells were centrifuged $\left(100 \times \mathrm{g}, 3 \mathrm{~min}, 4^{\circ} \mathrm{C}\right)$ and homogenized with 3 volumes of freshly made buffer A with a Dounce homogeniser. Homogenates were centrifuged at $105,000 \times \mathrm{g}$ for $1 \mathrm{~h}$, at $4^{\circ} \mathrm{C}$. High speed supernatants were used as cytosolic fractions and kept frozen $\left(-70^{\circ} \mathrm{C}\right)$ for further experiments.

\section{Preparation of cellular extracts}

For the determination of ezrin expression, whole cellular extracts were prepared additionally. Confluent cells were washed twice with PBS, scraped with a rubber policeman, suspended in PBS and centrifuged $(2,600 \times \mathrm{g}, 5$ min, $4^{\circ} \mathrm{C}$ ). This step was repeated twice. Then the cell pellets were treated with TET buffer $1 \mathrm{mM}$ Tris-HCl pH 8.0; $20 \mathrm{mM}$ EDTA; 0.5\% Triton X-100 with proteinase inhibitors: $1 \mathrm{mM}$ AEBSF (4-(2-Aminoethyl) benzenesulfonyl fluoride hydrochloride), $0.8 \mu \mathrm{M}$ aprotinin, $0.02 \mu \mathrm{M}$ leupeptin, $0.04 \mu \mathrm{M}$ bestatin, $0.15 \mu \mathrm{M}$ pepstatin and $0.14 \mu \mathrm{M}$ E-64. Finally the cells were sonicated, centrifuged $(2,000 \times \mathrm{g}, 30 \mathrm{sec}$, $4^{\circ} \mathrm{C}$ ) and the supernatants were used in Western blotting experiments for ezrin identification.

\section{Electrophoretic procedures}

Cytosolic fractions and in addition cellular extracts (in the case of ezrin) were used in electrophoretic and Western blotting experiments. Protein concentration was determined by the standard Bradford ${ }^{36}$ procedure. Identical amounts of protein $(30 \mu \mathrm{g})$ were subjected to analysis. Proteins were separated on polyacrylamide gels according to Laemmli ${ }^{37}$ and transferred to nitrocellulose sheets using a semi-dry blotter. ${ }^{38}$ Nitrocellulose sheets were incubated with $3 \%$ fish gelatine (Serva, Heidelberg, Germany) in Tris-buffered saline (TBS) and $0.05 \%$ Tween 20 (TBS-T) for $1 \mathrm{~h}$ at room temperature and blocked with $3 \%$ skimmed milk powder at $4^{\circ} \mathrm{C}$ in TBS-T overnight. The blots were incubated with TBS-T containing 5\% skimmed milk powder with the antibody directed against a selected ABPs (see below) and incubated overnight at $4^{\circ} \mathrm{C}$. Then the membranes were washed 3 times in TBS-T, followed 
by an incubation with horseradish peroxidaseconjugated goat anti-rabbit or goat anti-mouse antibodies (1:2,000). The enhanced chemiluminescence (ECL) system (Amersham, Germany) was used to develop the blots with the same exposure time for each antigen.

\section{Antibodies}

The monoclonal mouse anti-ezrin antibody was obtained from Sigma (No E 8897, clone $3 \mathrm{C} 12$, Munich, Germany). The polyclonal rabbit anti-cofilin antibody was raised in rabbits against recombinant human cofilin or cofilin:actin complex. The antibody was purified on Sepharose-cofilin affinity columns, then the antibody specificity was tested by Western blotting using purified human recombinant cofilin. The results showed that the anti-cofilin antibody was monospecific. ${ }^{39}$ The polyclonal rabbit anti-phosphorylated-cofilin (Ser3) antibodies were obtained from Cell Signalling (No 3311, Frankfurt, Germany). $\beta$ tubulin, labelled by mouse monoclonal anti- $\beta$ tubulin antibody (Sigma-Aldrich), was used as internal loading control during all Western blotting experiments.

\section{Densitometric analysis}

The intensity of bands interacting with antibodies was quantified by densitometry, using the software Bio-1-D. The values of cofilin, Pcofilin and ezrin expression obtained for colon adenocarcinoma cell variants were compared with the staining intensity measured for NRK and parental LS180 cells. Bars in figures represent the mean from three independent experiments for each cell line, each performed in triplicate $( \pm$ SD). Significance of difference was calculated with Student's t-test at $\mathrm{P}<0.05$.

\section{Fluorescence microscopy}

Actin microfilament organization and the subcellular distribution of the ABPs were observed with application of confocal laser scanning fluorescence microscope Zeiss LSM 510 (Jena, Germany). The cells growing on coverslips $(24 \mathrm{~h})$ were fixed with $4 \%$ paraformaldehyde in PBS for 20 min at room temperature and then permeabilized with ice-cold methanol or $0.1 \%$ Triton X-100 for 6 min and subsequently washed three times in PBS. After blocking with $1 \%$ bovine serum albumin (BSA) in PBS for $30 \mathrm{~min}$, the coverslips were incubated with antibodies directed against cofilin, Pcofilin and ezrin for 1 hour at room temperature. Then the coverslips were washed three times in PBS and incubated with goat antimouse IgG or goat anti-rabbit IgG conjugated with Alexa Fluor 488 (diluted 1:200 in 1\% BSA in PBS). After washing in PBS (3x) the actin microfilaments were visualized by staining with TRITC-conjugated phalloidin (Sigma,

(A)

Migration assay

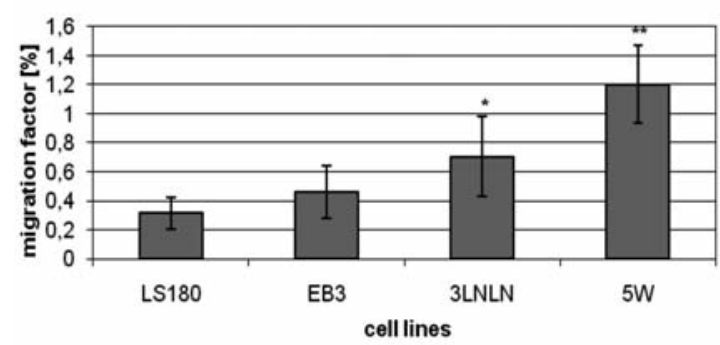

(B)

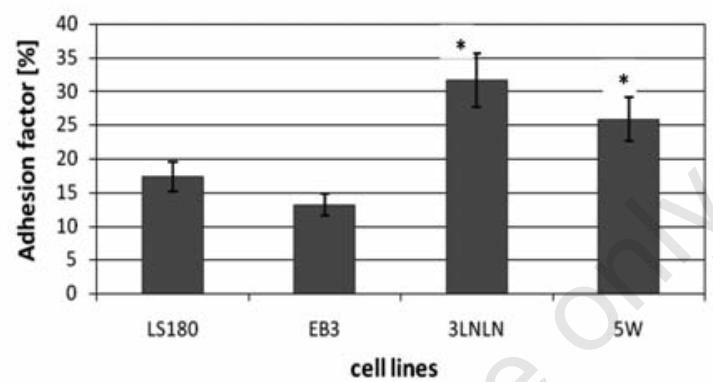

(A)

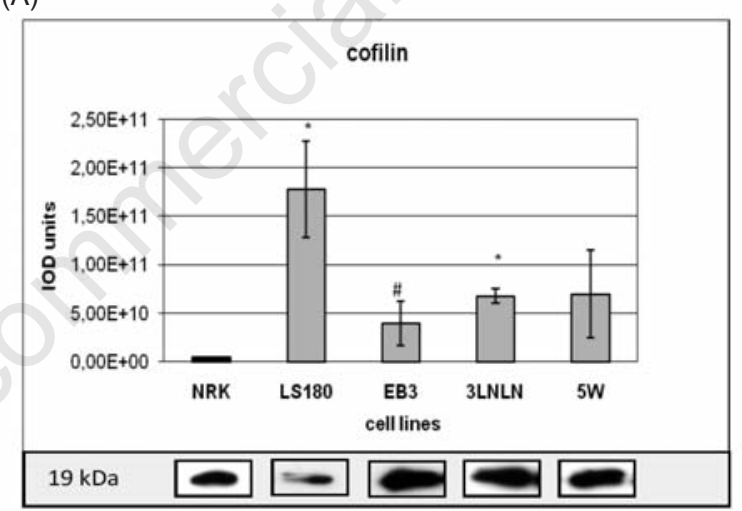

(B)

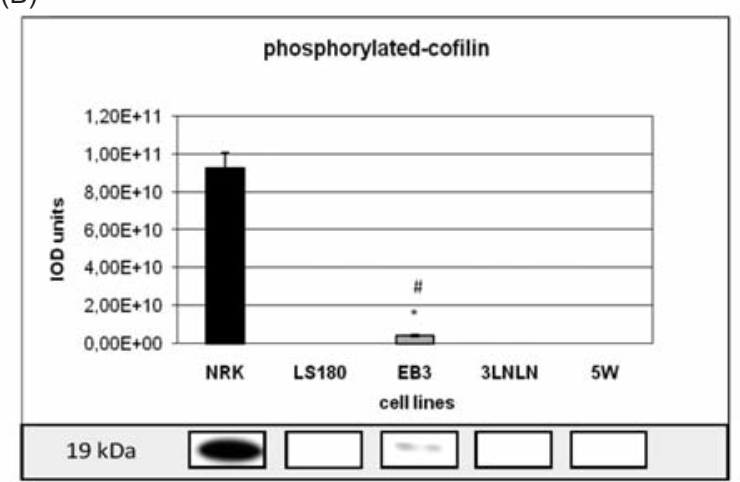

(C)

loading control $-\beta$ tubulin

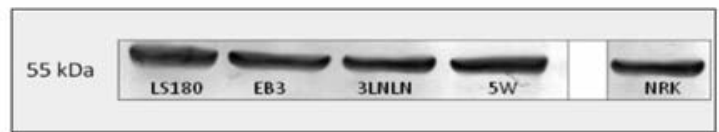

Figure 1. Migration (A) and adhesion (B) abilities of parental and selected human colon adenocarcinoma sublines. (A) Migration factor [\%] - the number of cells which migrate through Transwell filters $(24 \mathrm{~h})$ to the total number of cells seeded. The experiment was performed three times in nine repeats per every cell line. (B) Adhesion factor [\%] - the number of cells which adhere to plastic surface of culture dish after 3 hours to the total number of cells seeded. The experiment was performed three times per every cell line. The statistical significance of the differences was confirmed by Student's t-test, $\mathbf{P}<0.05 ; \quad(*), \quad \mathbf{P}<0.01 ; \quad(* *)$ indicates values significantly different for selected human adenocarcinoma sublines in comparison to parental LS180 cells.
Figure 2. The level of cofilin (A) and P-cofilin (B) in the cytosolic fractions from colon adenocarcinoma cell variants and normal NRK cells. SDS/PAGE and Western blotting with antibodies recognizing the examined proteins (see Materials and Methods) followed by densitometric analysis were used. Thirty $\mu \mathrm{g}$ of protein was applied in each case. Bars represent the mean from three independent experiments, each performed in triplicate $( \pm$ SD) Results are expressed as units of integrated optical density (IOD). The statistical significance of the differences was confirmed by Student's t-test, $\quad \mathbf{P}<\mathbf{0 . 0 5}$;

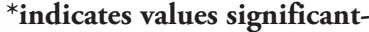
ly different from those for normal NRK cells; \#indicates values significantly different for human adenocarcinoma sublines in comparison with parental LS180 cells. The examples of a single representative immunoblotting reaction for each cell line are shown in the inserts. $\beta$-tubulin was used as the loading control during all Western blotting experiments (representative immunoblot - C). 
Munich, Germany) employed at a dilution of 1:200 (for $15 \mathrm{~min}$ in darkness). About 25 cells were photographed every time in three independent experiments and representative cells of every subline are presented.

\section{Results}

\section{Cells migratory and adhesive properties}

Well characterized phenotypically human colon adenocarcinoma LS180 parental cell line and its selected in vitro (EB3) or in vivo (3LNLN, 5W) sublines with different metastatic potential were used. ${ }^{30,31}$ Their migration ability was checked directly before experiments in modified Transwell ${ }^{\circledR}$ migration assay (see Materials and Methods). Significant migration factor (two fold higher migration factor for 3LNLN and three-fold higher for $5 \mathrm{~W}$ cells) was observed. Only slight increase in migration factor value was presented by EB3 subline (Figure 1A). Furthermore distinct differences in cell adhesion to plastic surface of culture dish were noticed. In vivo selected sublines (especially 3LNLN) have shown higher adhesiveness in reference to parental LS180 cells and in vitro selected subline (Figure 1B). Migratory and adhesive properties of human colon adenocarcinoma variants make them interesting model to focus on cofilin and ezrin - proteins important in the regulation of actin polymerization, cell migration and adhesion. Fibroblastic cells (NRK) were chosen as control, because they possess a well developed actin cytoskeleton and firmly attach to the substratum after spreading. The data obtained for the more motile colon adenocarcinoma cell variants were compared with parental LS180 and fibroblastic NRK cells.

\section{Cofilin}

Cofilin is an essential protein that controls cytoskeleton dynamics at the leading edge of motile cells particularly by increasing the speed of actin treadmilling process. ${ }^{910,40}$ The polyclonal anti-cofilin antibody was used to estimate the level of cofilin in the cytosolic fractions of investigated cells. A much higher level of total cofilin in the parental LS180 cells and the selected sublines was observed when compared with NRK cells. However, the cofilin expression appeared lower in the highly metastatic sublines (EB3, 3LNLN, 5W) than in the parental line (Figure $2 \mathrm{~A}$ ).

The actin binding activity of cofilin is inhibited by phosphorylation of Ser3..$^{14,15}$ In order to determine the fraction of inactivated cofilin in the different cell lines antibody directed against phosphorylated P-cofilin, Western blot-
Anti-cofilin antibodies (left); Phalloidin-TRITC (middle), Merge (right);
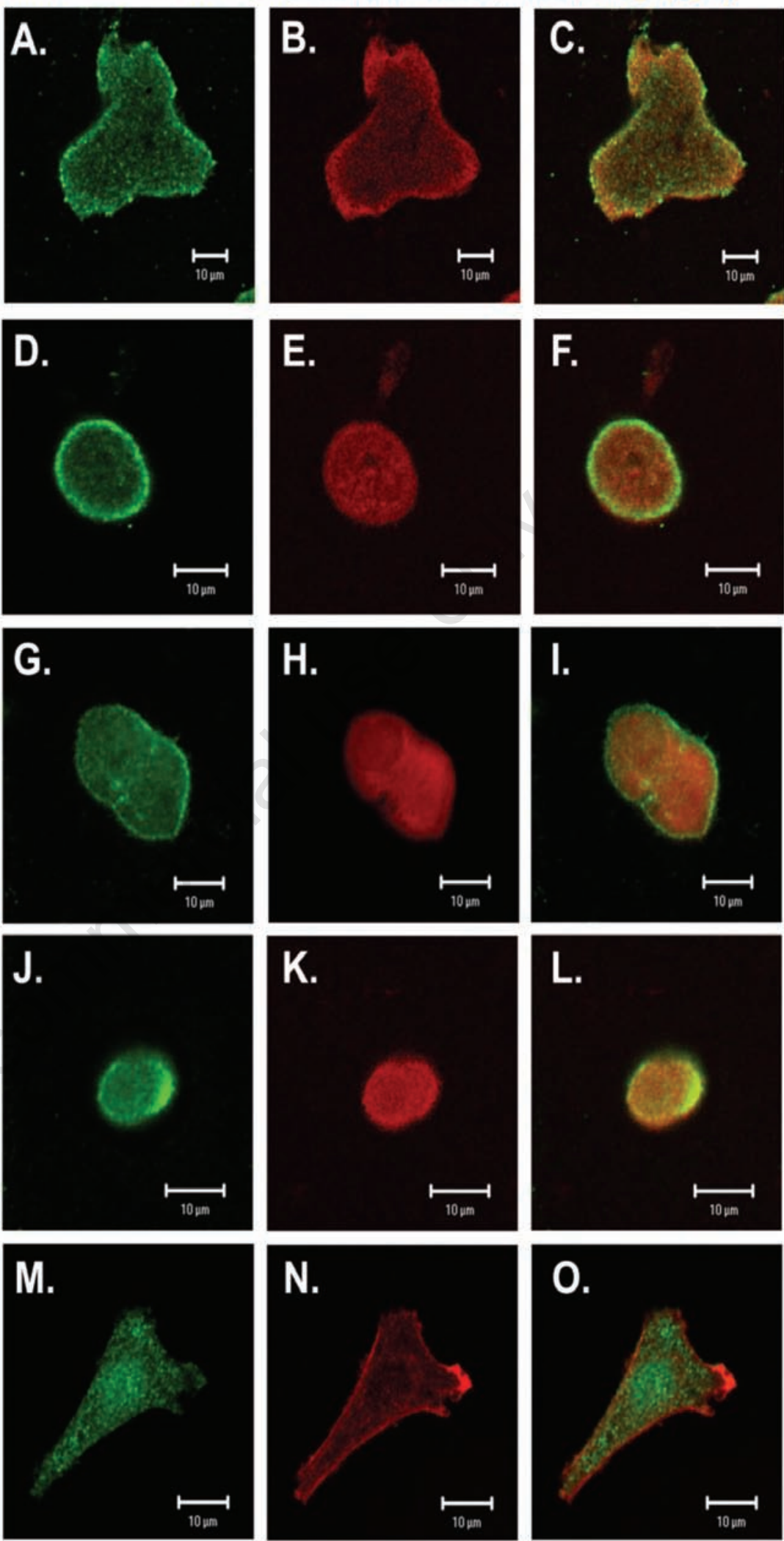

Figure 3. Subcellular distribution of cofilin in colon adenocarcinoma cells (A-C) Parental LS180 cells; (D-F) EB3; (G-I) 3LNLN; (J-L) 5W and (M-O) NRK cells. Scale bar: $10 \mu \mathrm{m}$. Left panel (green): immunostaining with antibodies against cofilin conjugated with Alexa Fluor 488; middle panel (red): actin - visualized by TRITC conjugated phalloidin; right panel: merge (green + red). 
ting and densitometry were applied. Interestingly, an inverse relation for inactive, phoshorylated cofilin (Figure 2B) in comparison with total cofilin (Figure 2A) was seen. Thus, a very high level of P-cofilin was observed in NRK cells in contrary to all adenocarcinoma variants, where strongly reduced relative amounts of P-cofilin were found. It was remarkably decreased in EB3 and almost not detectable in LS180, 3LNLN and 5W cells under the applied assay conditions (Figure 2B).

Next confocal microscopy was used to observe cofilin localization in adenocarcinoma and NRK cells. Colon adenocarcinoma cells (specially EB3 and 5W) often grow in clusters. It is difficult to catch images showing the real localization and organization of ABPs with high resolution and for this reason usually single cells were photographed. The results of subcellular distribution of cofilin and P-cofilin in human colon adenocarcinoma and NRK cells are presented in Figure 3 and Figure 4. Clear differences among cells characterized by diverse metastatic potential were observed. In parental adenocarcinoma LS180 and normal NRK cells, cofilin was dispersed in the whole cell body with a visible accumulation at the cell leading edge (Figure $3 \mathrm{~A}, \mathrm{C}$ and $\mathrm{M}, 0$ ). In the selected, more motile variants of colon adenocarcinoma (EB3, 3LNLN and 5W), cofilin was localized mainly at the peripheral region of the cells. In addition, the highest concentration of this protein was observed in the most dynamic cellular area, i.e. under the cellular membrane known as 'cortical ring' (Figure $3 \mathrm{D}, \mathrm{G}, \mathrm{J}$ and F,I,L). This characteristic distribution was particularly well seen in EB3 cells (Figure 3 D,F). In contrast, phosphorylated, inactive cofilin was visualized in the center of cell bodies of the parental adenocarcinoma LS180 cells, the selected more motile variants and the NRK cells (Figure 4). An intensive reaction in the region of nucleus was observed in EB3 cells (Figure 4 D,F).

\section{Ezrin}

Ezrin is a member of the ERM family, which belongs to the band 4.1 of membrane proteins. Immunolocalization and a Western blots analysis of ezrin were performed in both the soluble, i.e. the cytoplasmic fraction (supernatant $105,000 \times \mathrm{g}$ ) and the whole cellular extracts. The latter fraction also includes ezrin firmly bound to cellular membranes. The densitometric analysis showed the highest level of ezrin presented in the cytoplasm of the parental adenocarcinoma LS180 and NRK cells, whereas relatively low levels of ezrin were seen in the cytosolic fractions of the invasive sublines (EB3, 3LNLN and 5W) (Figure $6 \mathrm{~A}$ ).

Western blot analysis was repeated on extracts obtained after homogenization of the

Anti-P-cofilin antibodies (left); Phalloidin-TRITC (middle), Merge (right);
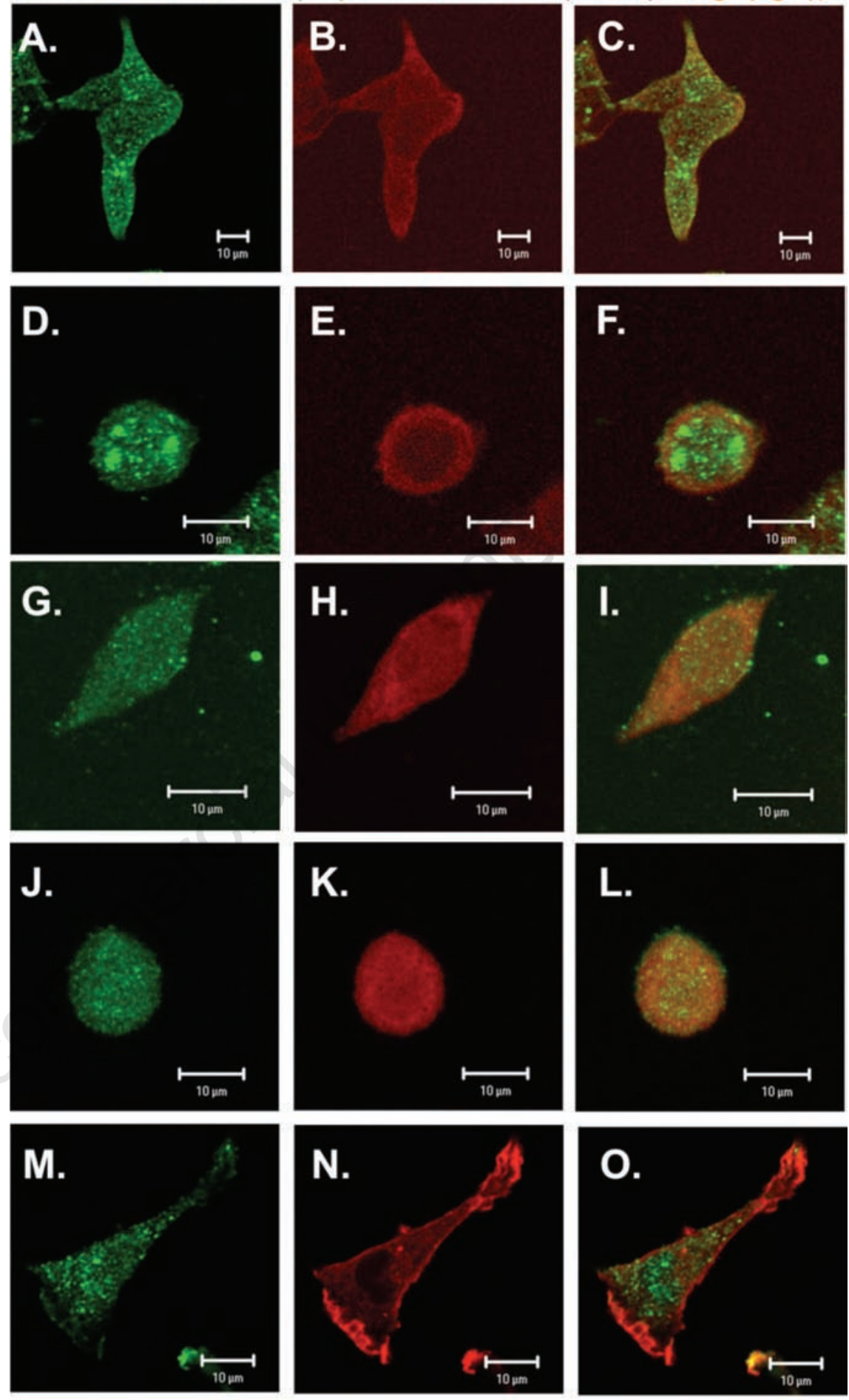

Figure 4. Phosphorylated (inactive) cofilin in colon adenocarcinoma cells. (A-C) Parental LS180 cells; (D-F) EB3; (G-I) 3LNLN; (J-L) 5W and (M-O) NRK cells. Scale bar: 10 $\mu \mathrm{m}$. Left panel (green): immunostaining with antibodies against P-cofilin conjugated with Alexa Fluor 488; middle panel (red): actin - visualized by TRITC-conjugated phalloidin; (right panel) merge.

cells in Triton X-100 containing buffer (see Material and Methods) to detect the ezrin firmly bound to membranes. The subsequent quantitative densitometric analysis demonstrated that there were considerably higher levels of ezrin in the cell extracts of invasive EB3, $3 \mathrm{LNLN}$ and $5 \mathrm{~W}$ sublines, in contrast with the parental LS180 line (compare Figure 6 A,B, see values identified by ${ }^{*}$ and \#). We assume that this result indicates a translocation of ezrin to 
the plasma membrane. The immunolocalization of ezrin with monoclonal antibodies against ezrin supports this assumption. In elongated, well spread cells such as NRK and LS180, ezrin was distributed in the whole cell body and localized at the tips of pseudopodia (Figure $5 \mathrm{~A}, \mathrm{C}$ and. $\mathrm{M}, 0$ ). On the contrary, in selected, rounded colon adenocarcinoma EB3 and $5 \mathrm{~W}$ sublines this protein was localized as a sharp ring under the cellular membrane (Figure 5 D,F,J,L). In 3LNLN cells, ezrin was present at the cell edges in areas of subtle protrusions (Figure $5 \mathrm{G}, \mathrm{I}$ ). Thereby, our data seem to indicate an increase in ezrin in close connection with plasma membrane in the human colon adenocarcinoma sublines with higher invasive potential. In summary, we conclude that there are distinct differences in the subcellular localization of ezrin in the more motile colon adenocarcinoma cells in comparison with the parental LS180 line as well as to NRK cells.

\section{Discussion}

Directional movement of cells plays an important role in many physiological processes, such as embryogenesis, wound healing, angiogenesis and host defense against pathogens. Under pathological conditions, an increased motility of tumor cells leads to metastasis and the life-threatening invasion of distant organs. ${ }^{2} \mathrm{~A}$ better understanding of the molecular mechanisms of cell locomotion during these physiological and pathological events is therefore of high clinical relevance.

In this study a few cell sublines derived from a human colon adenocarcinoma were used. Human tumors are usually composed by cells with different invasive potential. For this reason the established parental adenocarcinoma line had previously been fractionated into a number of sublines of different invasive behaviour by in vitro and in vivo selection procedures. $^{32,33}$ Selected sublines were characterized as significantly different on account of migration and adhesion abilities. The expression and distribution of two actin binding proteins, cofilin and ezrin, were analyzed in these human colon adenocarcinoma cells. These proteins are essentially involved in cell migration and adhesion. Our data clearly demonstrate a distinct upregulation of cofilin in tumor cells accompanied by the reduction of the inactive phosphorylated form of cofilin. The presented data are in agreement with previously published results, which have shown over-expression of cofilin in the highly invasive $\mathrm{C} 6$ rat glioblastoma cell line and in cells derived from human breast cancer. ${ }^{41-44}$ Furthermore, the decreased level of phosphorylated, inactive

\section{Anti-ezrin antibodies (left); Phalloidin-TRITC (middle), Merge (right);}
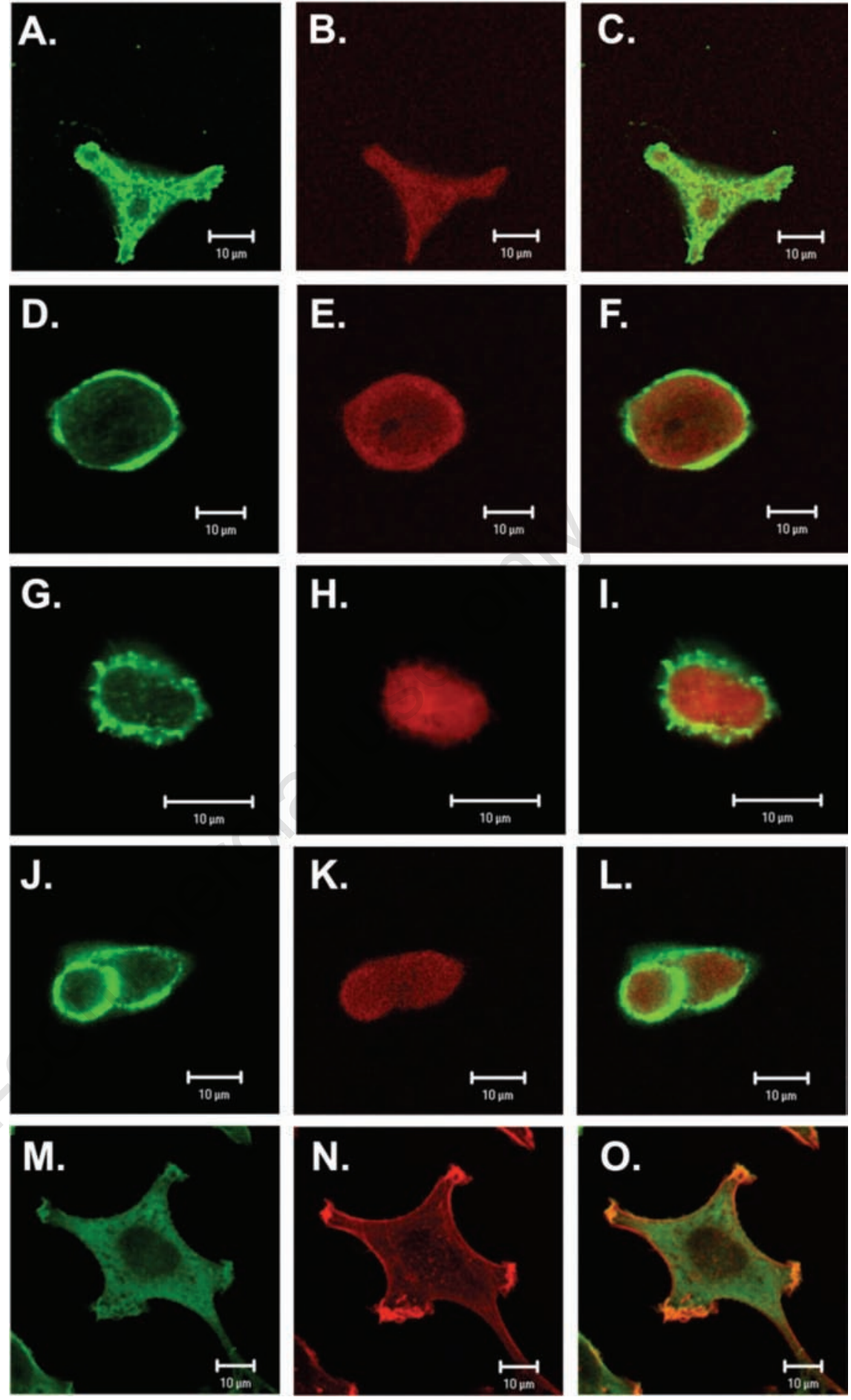

Figure 5. Ezrin location in colon adenocarcinoma sublines with different metastatic potential. (A-C) parental LS180 cells; (D-F) EB3; (G-I) 3LNLN; (J-L) 5W and (M-O) NRK cells. Scale bar: $10 \mu \mathrm{m}$. Left panel (green): immunostaining with antibodies against ezrin conjugated with Alexa Fluor 488; middle panel (red): actin - visualized by TRITCconjugated phalloidin; right panel: merge.

cofilin was reported for T-cell lymphoma, cervix carcinoma (HeLa), colon cancer (KM12), liver (HepG2), and kidney (COS1) cells. ${ }^{4446}$ Our results also show a similar correlation of cofilin expression and activation, i.e. the decrease of the phosphorylated form in tumor cell lines with high invasive potential. We demonstrate that this correlation is independent of tissue variations, since we used cells selected from a single parental adenocar- 
(A)

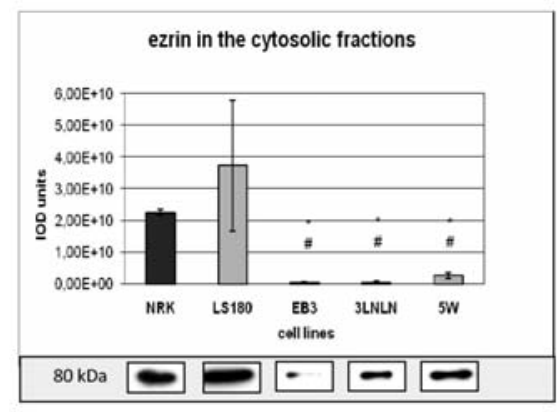

(B)

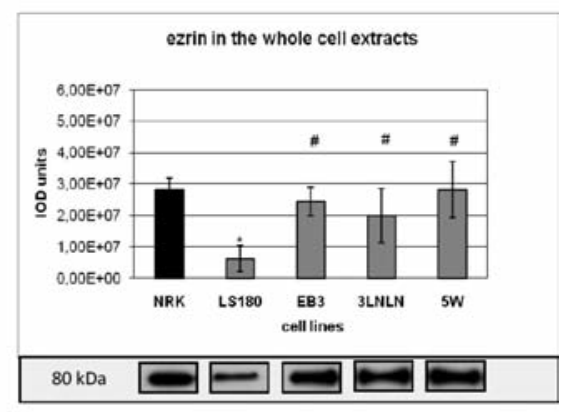

Figure 6. Ezrin in the cytosolic fraction (A) and in the whole cellular extracts (B) of colon adenocarcinoma cell variants and normal NRK cells. Bars represent the mean from three independent experiments each performed in triplicate $( \pm$ SD). Results are expressed as units of integrated optical density (IOD). The statistical significance of the difference was confirmed by Student's t-test, $\mathbf{P}<\mathbf{0 . 0 5}$; ${ }^{*}$ indicates values significantly different from those for NRK cells; \#indicates values significantly different for human adenocarcinoma sublines in comparison to parental LS180 cells. $\beta$-tubulin was used as the loading control during all Western blotting experiments.

cinoma line. In more motile, rounded colon adenocarcinoma sublines, cofilin was accumulated at their periphery in co-localization with actin filaments. Inactive cofilin was mainly concentrated in the perinuclear region of $\mathrm{NRK}^{39}$ and parental LS180 cells, but strongly decreased in the invasive adenocarcinoma cells. Hence, our data indicate that also in human colon adenocarcinoma sublines, cofilin may play an important role in the dynamic turnover of actin filaments and therefore may be essentially involved in the higher migratory activity of these invasive tumor cells.

Previous reports demonstrated that ezrin was over-expressed in mouse sarcoma, human endometrial, pancreatic and colorectal carcinomas, gliomas and uveal melanoma. ${ }^{26,27}$

We have shown a significant increase in the level of ezrin expression in selected adenocarcinoma cells with higher migratory ability (EB3, 3LNLN, 5W) and higher adhesive properties (3LNLN and $5 \mathrm{~W}$ ). In addition, our data indicate a clear translocation of ezrin from cytoplasm towards the cell periphery within the highly motile adenocarcinoma cells, whereas very low amounts were seen by Western blotting in the cytosolic fractions isolated from these invasive sublines. In contrast, the percentage of ezrin within the cytosol was much higher in NRK and the parental LS180 adenocarcinoma cells than in EB3, 3LNLN and $5 \mathrm{~W}$ cells. The same submembranous ezrin localization was demonstrated by the microscopic studies in selected human adenocarcinoma sublines. Thus, ezrin translocation to the plasma membrane was previously suggested to occur in tumor cells. ${ }^{21,23,47,48}$ This effect could be connected with the phosphorylation of ezrin, as previous data indeed have shown that phosphorylation of ezrin induces its activation and translocation from the cytoplasm to the plasma membrane of microvilli. Presumably, this process is under control of the Rho-ROCK kinase. ${ }^{21,23,48}$ Active ezrin is believed to attach actin filaments to the plasma membrane and it can change adhesive properties of cells. Consequently, the membranous ezrin localization may induce alteration in ezrin-mediated cellular processes. Ezrin is activated through stimulation by external growth factors, giving an open conformation able to link actin and transmembrane receptors. ${ }^{47}$ Such a phenomenon is commonly observed after EGF stimulation. This effect is particularly clear in case of EB3 cells selected in vitro by means of migration towards endothelial cells releasing great amounts of growth factors. The re-distribution of ezrin from cytosol to microvilli and membrane ruffles under the influence of EGF was demonstrated in variety of cancer cell lines..$^{24}$ Hence, in selected highly motile (EB3, 3LNLN and 5W) and adhesive cells (3LNLN, $5 \mathrm{~W}$ ), ezrin was mainly membranous. The increase of ezrin close to the plasma membranes in our case correlates with enhanced invasion, migration and adhesion in vitro. A number of authors have suggested that ezrin was required for tumor cell migration and metastasis formation. ${ }^{18,28,48}$ This protein has been shown to be important for cell adhesion and motility. The C-terminus of active, i.e. phosphorylated ezrin, associates to actin filaments, whereas its $\mathrm{N}$-terminus binds to integral plasmalemmal adhesion molecules such as CD43, CD44, ICAM-1 or ICAM-2. ${ }^{16,23,48}$ Anchoring of Factin to adhesion molecules of the cell surface by ezrin may be a necessary requisite for cell migration during metastasis. Indeed, high CD44 expression was also found to be associated with invasive and metastatic behaviour of tumor cells. ${ }^{48,49}$ However, selected human adenocarcinoma sublines do not display any changes in the expression and distribution in CD44 level (data not shown). Supposedly, it may be due to the fact that this molecule, important for cell adhesion, is also a marker of colon adenocarcinoma and that parental line has already a high expression of this protein.

In summary, our data demonstrate a clear correlation between the metastatic potential of human colon adenocarcinoma cells and different expression and peripheral localization of cofilin and ezrin. It would be interesting to specify details of this phenomenon and investigate if the activity of both proteins are regulated by Rho-ROCK kinase in this particular example. The activation of this kinase has been shown to occur in a particular form of motility (the amoeboid migration) that is frequently observed for tumor cells when they transmigrate the fibrillar network of a dense extracellular matrix., ${ }^{42,44,50.53}$ Very little is presently known about the mechanism of amoeboid movement that is characterized by the formation of round plasma membrane extensions (blebs) at the leading front of migrating cells. ${ }^{54}$

In the future it would be interesting to analyze the regulation of biological activity of cofilin and ezrin in amoeboidal type of cancer cells movement.

\section{References}

1. Horwitz R, Webb D. Cell migration. Curr Biol 2003;13:R756-9.

2. Kassis J, Lauffenburger DA, Turner T, Wells A. Tumour invasion as dysregulated cell motility. Semin Cancer Biol 2001;11: 105-17.

3. Sheterline P, Clayton J, Sparrow JC. Protein profile. Actin. New York: Oxford University Press; 1998.

4. Lambrechts A, Van Troys M, Ampe C. The actin cytoskeleton in normal and pathological motility. Int J Biochem Cell Biol 2004; 36:1890-1909.

5. Hall A. Rho GTPases and the control of cell behaviour. Biochem Soc Trans 2005;33: 891-95.

6. Pantaloni M, Le Clainche C, Carlier MF. Mechanism of actin-based motility. Science 2001;292:1502-6.

7. Carlier MF, Laurent V, Santolini J , Melki R, Didry D, Xia G et al. Actin Depolymerizing Factor (ADF/Cofilin) enhances the rate of filament turnover: implication in actin-based motility. J Cell Biol 1997;136:1307-23.

8. Chen H, Bernstein BW, Bamburg JR. Regulating actin-filament dynamics in vivo. Trends Biochem Sci 2000;25:19-23.

9. Condeelis J. How is actin polymerization nucleated in vivo? Trends Cell Biol 2001; 11:288-93.

10. Ghosh M, Song X, Mouneimne G, Sidani M, Lawrenc DS, Condeelis J. Cofilin promotes actin polymerization and defines the direction of cell motility. Science 2004; 304:743-8.

11. Gurniak CB, Perlas E, Witke W. The actin depolymerizing factor $n$-cofilin is essential 
for neural tube morphogenesis and neural crest migration. Dev Biol 2005;278:231-41.

12. Andrianantoandro E, Pollard TD. Mechanism of actin filament turnover by severing and nucleation at different concentrations of ADF/cofilin. Mol Cell 2006;24:13-23.

13. Pavlov D, Muhlrad A, Cooper J, Wear M, Reisler E. Actin filament severing by cofilin. J Mol Biol 2007;365:1350-8.

14. Mizuno K, Okano I, Ohashi K, Nunoue K, Kuma K, Miyata $\mathrm{T}$ et al. Identification of human cDNA encoding a novel protein kinase with two repeats of the LIM/double zinc finger motif. Onco-gene 1994;9:1605-12.

15. Okano I, Hiraoka J, Otera H, Nunoue K, Ohashi K, Iwashita $S$ et al. Identification and characterization of a novel family of serine/threonine kinases containing two $\mathrm{N}$ terminal LIM motifs. J Biol Chem 1995;270: 31321-30.

16. Gautreau A, Poullet P, Louvard D, Arpin M. Ezrin, a plasma membrane- microfilament linker, signals cell survival through the phosphatidilinositol 3-kinase/Akt pathway. Proc Natl Acad Sci (USA) 1999;96:7300-5.

17. Mangeat P, Roy C, Martin M. ERM proteins in cell adhesion and membrane dynamics. Trends Cell Biol 1999;9:187-92.

18. Elliot BE, Meens JA, SenGupta S, Louvard D, Arpin M. The membrane cytoskeletal crosslinker ezrin is required for metastasis of breast carcinoma. Breast Cancer Res 2005;7:R365-73.

19. Fievet B, Louvard D, Arpin M. ERM proteins in epithelial cell organization and functions. Biochim Biophys Acta 2007; 1773:653-60.

20. Bretscher A, Edwards K, Fehon RG. ERM proteins and merlin: integrators at the cell cortex. Nat Rev Mol Cell Biol 2002;3:586-99.

21. Yonemura S, Matsui T, Tsukita S, Tsukita S. Rho-dependent and -independent activation mechanisms of ezrin/radixin/moesin proteins: an essential role for polyphosphoinositides in vivo. J Cell Sci 2002;115:2569-80.

22. Sahai E, Marshall CJ. Differing modes of tumour cell invasion have distinct requirements for Rho/ROCK signaling and extracellular proteolysis. Nat Cell Biol 2003; 5:711-9.

23. Gautreau A, Louvard D, Arpin M. ERM proteins and NF2 tumor suppressor: the Yin and Yang of cortical actin organization and cell growth signaling. Curr Opin Cell Biol 2002;14:104-9.

24. Hiscox S, Jiang WG. Ezrin regulates cell-cell and cell-matrix adhesion, a possible role with E-cadherin/ $\beta$-catenin. J Cell Sci 1999; 112:3081-90.

25. Ohtani K, Sakamoto H, Rutherford T, Chen Z, Satoh K, Naftolin F. Ezrin, a membranecytoskeletal linking protein, is involved in the process of invasion of endometrial cancer cells. Cancer Lett 1999;147:31-8.

26. Geiger KD, Stoldt P, Schlote W, Derouiche A.
Ezrin immunoreactivity is associated with increasing malignancy of astocytic tumors but is absent in oligodendro-gliomas. Am J Pathol 2000;157:1785-93.

27. Mäkite T, Carpen 0, Vaheri A, Kivelä T. Ezrin as a prognostic indicator and its relationship to tumor characteristic in uveal malignant melanoma. Invest Ophthalmol Vis Sci 2001;42:2442-9.

28. Rossy J, Gutjahr MC, Blaser N, Schlicht D, Niggli V. Ezrin/moesin in motile Walker 256 carcinosarcoma cells: signal-dependent relocalization and role in migration. Exp Cell Res 2007;313:1106-20.

29. Kanna C, Wan X, Bose S, Cassaday R, Olomu 0 , Mendoza A et al. The membrane-cytoskeleton linker ezrin is necessary for osteosarcoma metastasis. Nat Med 2004;10: 182-6.

30. Nowak D, Krawczenko A, Duś D, MalickaBłaszkiewicz M. Actin in human colon adenocarcinoma cells with different metastatic potential. Acta Biochim Polon 2002;49:823-8.

31. Nowak D, Skwarek-Maruszewska A, Zemanek-Zboch M, Malicka-Błaszkiewicz M. Betaactin in human colon adenocarcinoma cell lines with different metastatic potential. Acta Biochim Polon 2005;52:461-8.

32. Opolski A, Wietrzyk J, Duś D, Kieda C, Matejuk A, Makowska A et al. Metastatic potential and saccharide antigens expression of human colon cancer cells xenotransplanted into athymic nude mice. Folia Microbiol 1998;43:507-10.

33. Kieda C, Paprocka M, Krawczenko A, Załecki P, Dupuis P, Monsigny M et al. New human microvascular endothelial cell lines with specific adhesion molecules phenotypes. Endothelium 2002;9:247-61.

34. Humphries MJ. Cell adhesion assay. Mol Biotechnol 2001;18:57-61.

35. Malicka-Błaszkiewicz M, Roth JS. Some factors affecting the interaction between actin in leukemic L1210 cells and DNase I. Biochem Biophys Res Commun 1981;102: 594-601.

36. Bradford MM. A rapid and sensitive method for the quantitation of microgram quantities of protein utilizing, the principle of proteindye binding. Anal Biochem 1976;72: 248-54.

37. Laemmli UK. Cleavage of structural proteins during the assembly of the head of bacteriophage T4. Nature 1970;227:680-5.

38. Towbin H, Stachelin T, Gordon T. Electrophoretic transfer of proteins from polyacrylamide gels to nitrocellulose sheets: procedure and some applications. Proc Natl Acad Sci USA 1979;76:4350-4.

39. Mannherz HG, Gonsior SM, Wu X, Polzar B, Pope BJ, Wartosch L et al. Dual effects of Staurosporine on A431 and NRK cells: Microfilament disassembly and uncoordinated lamellipodial activity followed by cell death.
Eur J Cell Biol 2006; 85:785-802.

40. McGough A, Pope B, Weeds AG. Molecular Interactions of Actin: Actin-Binding Proteins. vol 32; Heidelberg, Germany: SpringerVerlag 2001.

41. Gunnersen JM, Spirkoska V, Smith PE, Danks RA, Tan SS. Growth and migration markers of rat $\mathrm{C} 6$ glioma cells identified by serial analysis of gene expression. Glia 2000;32:146-54.

42. Wang W, Goswami S, Lapidus K, Wells AL, Wyckoff E, Sahai E et al. Identification and testing of a gene expression signature of invasive carcinoma cells within primary mammary tumors. Cancer Res 2004;64:8585-94.

43. Wang W, Eddy R, Condeelis J. The cofilin pathway in breast cancer invasion and metastasis. Nature Rev (Cancer) 2007; 7:429-40.

44. Yamaguchi H, Condeelis J. Regulation of actin cytoskeleton in cancer cell migration and invasion. Biochim Biophys Acta 2007; 1773:642-52.

45. Nebl G, Meuer SC, Samstag Y. Dephosphorylation of serine 3 regulates nuclear translocation of cofilin. J Biol Chem 1996;271:26276-80.

46. Subramaniam V, Vincent IR, Jothy S. Upregulation and dephosporylation of cofilin: modulation by CD44 variant isoforms in human colon cancer cells. Exp Mol Pathol 2005;79:187-93.

47. Sarrio D, Rodriguez-Pinilla SM, Dotor A, Calero F, Hardisson D, Palacios J. Abnormal ezrin localization is associated with clinicopathological features in invasive breast carcinomas. Breast Cancer Res Treat 2006; 98:71-9.

48. Akisawa N, Nishimori I, Iwamura T, Onishi S, Hollingsworth MA. High levels of ezrin expressed by human pancreatic adenocarcinoma cell lines with high metastatic potential. Biochem Biophys Res Commun 1999;258:395-400.

49. Underhill C. Cd44: the hyaluronan receptor. J Cell Sci 1992;103:293-8.

50. Maekawa M, Ishizaki T, Boku S, Watanabe N, Fujita A, Iwamatsu A et al. Signaling from Rho to the actin cytoskeleton through protein kinases ROCK and LIM-kinase. Science 1999;285:895-8.

51. Sahai, E. Mechanisms of cancer cell invasion. Curr Opin Genet Develop 2005;15:87-96.

52. Vasiliew J. Cytoskeletal mechanism responsible for invasive migration of neoplastic cells. Int J Dev Biol 2004;48:425-39.

53. Wolf K, Friedl P. Molecular mechanisms of cancer cell invasion and plasticity. $\mathrm{Br} \mathrm{J}$ Dermatol 2006;154:11-5.

54. Mannherz HG, Mach M, Nowak D, MalickaBłaszkiewicz M, Mazur A. Lamellipodial and amoeboid cell locomotion: the role of actincycling and bleb formation. Biophys Rev Lett 2007;2:5-22. 